Original article

\title{
Prevalence and antimicrobial sensitivity pattern of bacteria causing urinary tract infection; study of a tertiary care hospital in North India
}

\author{
Gagan Chooramani, Bhawana Jain*, Prashant Singh Chauhan \\ Vivekananda Polyclinic and Institute of Medical Sciences, Lucknow, Uttar Pradesh, India
}

\section{A R T I C L E I N F O}

\section{Keywords:}

Urinary tract infection

Antimicrobial resistance

Tertiary care hospital

Epidemiology

Vitek

\begin{abstract}
A B S T R A C T
Background: Urinary Tract Infections (UTI) are one of the most common bacterial infections in routine clinical practice. It is also the most common nosocomial infection in many hospitals. Study of causative agents and their Antimicrobial sensitivity pattern are important factors helping formulating antibiotic policy. Generally, the most common uropathogen is Escherichia coli. Tertiary care hospitals, catering for already partially treated or maltreated patients as major chunk, may have different etiological agents and sensitivity pattern.

Objectives: To study prevalence of pathogens causing UTI among patients coming to a tertiary care hospital, along with their antibiotic sensitivity pattern.

Methods: This study was undertaken for a period from July 2017 to Nov'2018 at Department of Microbiology, Vivekananda Polyclinic Institute of Medical sciences, Lucknow, India. Clean catch mid-stream urine samples were collected from all suspected UTI patients of various departments. The urine samples were processed for culture and Antibiotic susceptibility testing according to standard guidelines.

Results: A total of 10,171 urine samples were processed for urine culture and sensitivity, Total 1,728 samples were found to be positive for bacterial infection (amounting almost 17\% positivity). For the ease of analysis, positive isolates $(\mathrm{n}=1728$ ) were further categorized into isolates obtained from outdoor patients (OPD; $\mathrm{n}=824$ ), from indoor patients (IPD; $\mathrm{n}=747$ ) and from pediatric patients $(\mathrm{n}=157)$. Escherichia coli was the most frequently isolated urinary pathogen in all categories. Enterococcus and Candida were significantly isolated more in IPD patients.

Conclusion: Sensitivity pattern was also different in all categories. Tertiary care hospital has difference in prevalence of etiological agents and their sensitivity pattern.
\end{abstract}

\section{Introduction}

UrinaryTract Infection (UTIs) are one of the most common bacterial infection in routine clinical practice, clinical presentation of which ranging from asymptomatic to severe sepsis. ${ }^{1}$ UTI is one of the most important causes of morbidity in general population, and is the second most important cause of hospital visits. ${ }^{2}$ It also contributes as the most common nosocomial infection in many hospitals and accounts for approximately $35 \%$ of all hospital-acquired infections. ${ }^{3}$ This burden causes serious impact on the socioeconomic life of individuals and also leads to a large proportion of antibacterial drug consumption. ${ }^{4}$

Generally, the predominant uropathogens for UTIs are gram negative bacteria and Escherichia coli accounting for the highest prevalence in most instances. ${ }^{5}$ Other commonly involved urinary pathogens are Klebsiella spp., Proteus spp., Staphylococcus aureus, Enterobacter spp., Citrobacter spp., Pseudomonas aeruginosa, Acinetobacter spp., Enterococcus spp., Candida albicans. ${ }^{6}$ UTI is treated with broad spectrum antibiotics empirically to start with, which are de-escalated to specific antibiotic based on information obtained from the antimicrobial susceptibility pattern of the urinary pathogens. ${ }^{4}$ Widespread use of antimicrobial agents has lead to the emergence of antibiotic resistant pathogens; also there is increase demand for new drugs. Distribution of uropathogens and their antimicrobial sensitivity patterns may differ regionally so it becomes necessary to study these and compile their data in particular settings. ${ }^{7,8}$

Therefore, we have planned the study to analyse data of the organisms causing UTI and their antibiotic sensitivity patterns, from our hospital.This study was conducted in patients reporting to a tertiary health care center in Lucknow, Uttar Pradesh.

\footnotetext{
* Corresponding author.

E-mail addresses: gaganchooramani@gmail.com (G. Chooramani), drbhawana.goyal@gmail.com (B. Jain), prashant.chauhan012@gmail.com (P.S. Chauhan).
} 


\section{Materials and methods}

This study was undertaken for a period from July2017 to Nov.2018 at Department of Microbiology, Vivekananda Polyclinic Institute of Medical sciences, Lucknow, India. Clean catch mid-stream urine samples ${ }^{9}$ were collected from the all suspected UTI patients attending to OPD/IPD of various departments of Vivekananda Polyclinic Institute of Medical sciences, Lucknow, India, using sterile screw capped containers.

\subsection{Bacterial isolates}

The urine samples collected were examined microscopically for pus cells and casts and then were inoculated on Cysteine Lactose Electrolyte Deficient (CLED) agar medium. Inoculated agar plates were incubated initially aerobically at $37{ }^{\circ} \mathrm{C}$ for $24 \mathrm{~h}$ and finally for $48 \mathrm{~h}$. The urine culture plates were examined for pure growth. Next day individual colonies were primarily identified on the basis of colony morphology and gram staining. ${ }^{10}$ Further confirmation was done by VITEK 2 automated system (bioMerieux, Durham, North Carolina). ${ }^{10}$ Culture results were interpreted as being significant and not significant, according to the standard criteria. A growth of $\geq 10^{5}$ colony forming units $/ \mathrm{mL}$ was considered as significant bacteriuria. ${ }^{11}$ Patients with significant bacteriuria and symptomatic patients with lower bacteriuria were also considered. Cultures with more than two colonies were considered as contaminants and such samples were discarded.

\subsection{Antimicrobial susceptibility testing}

Antibiotic susceptibility tests and interpretations for the bacterial isolates were carried out by VITEK2 automated system (bioMerieux, Durham, North Carolina). Antibiotic resistance data were extracted from the hospital information system using software. The antimicrobial agents tested were as per Clinical and Laboratory Standards Institutes (CLSI) guidelines (M100-edition 28th) ${ }^{12}$ and The European Committee on Antimicrobial Susceptibility Testing (EUCAST) rules for each isolate.

Organisms resistant to more than three classes of drugs were considered as Multi Drug Resistant.

\section{Results}

During the study period (July'17 to Nov'18), the total number of samples sent to microbiology laboratory from out- and in-patients of the various departments were 12,663, of which 10,171 were for urine culture and sensitivity.Total 1,728 samples were found to be positive for urinary tract infection (amounting almost 17\% positivity). For the ease of analysis, positive isolates (Uropathogens; $n=1728$ ) were further categorized into isolates obtained from outdoor patients (OPD; $\mathrm{n}=824$ ), from indoor patients (IPD; $\mathrm{n}=747$ ) and from pediatric patients $(\mathrm{n}=157)$. (Table 1$)$.

Escherichia coli was the most frequently isolated urinary pathogen in all categories $(66.1 \% \mathrm{OPD}, 41.9 \%$ IPD and $42 \%$ pediatric patients). Enterococcus spp was the second in list, however its proportion varies significantly in different categories. It was isolated from $10.9 \%$ samples from OPD, while almost $30 \%$ of samples from IPD and pediatric population showed presence of Enterococcus spp. Klebsiella spp. was present in almost about $10 \%$ of samples of all categories. Candida spp and Acinetobacter spp. were more frequently isolated from IPD patients and pediatric patients. Proteus spp., Sphingomonas paucimobilis, Pseudomonas aeruginosa and Staphylococcus aureus were other bacteria isolated in varied proportions. (Table 1).

Antibiotic sensitivity patterns of most commonly isolated Gram negative and Gram-positive bacteria in different categories were studied and compared (Table 1, Figs. 1 and 2).

Table 2 showed that tigecycline shows highest sensitivity in Gram negative bacteria in all categories, followed by Fosfomycin, nitrofurantoin and carbapenem group. Piperacillin/Tazobactam shows significant reduction in sensitivity in case of Klebsiella isolates in comparison to E. coli. Cephalosporins, Tetracycline other than Tigecycline and Co-trimoxazole have low sensitivity for Gram Negative bacteria, in general. Fluoroquinolones showed least sensitivity in all categories (Table 2), particularly in IPD patients, it's nil.

Figure shows the difference in antibiotic sensitivity patterns among 3 categories, but that whole pattern is not statistically significant ( $\mathrm{p}$ value $=0.3595$ ).Tigecycline, Fosfomycin, Aminoglycosides, Nitrofurantoin shows approximately same sensitivity pattern in OPD, IPD \& pediatrics patients. While Carbapenem, Piperacillin/Tazobactam, Cephalosporin, Co-trimoxazole and Fluoroquinolones are more resistant in IPD patients as compared to OPD patients. Piperacillin/ Tazobactam and Fluoroquinolones are exhibiting poor sensitivity in adults, while they are still effective in pediatric patients.Piperacillin/ Tazobactam, Cephalosporin, Co-trimoxazole and Fluoroquinolones have almost completely lost their utility as $1^{\text {st }}$ line drugs in inpatients (sensitivity is $<30 \%$ ).

Enterococcus is the most common Gram-positive bacteria isolated in UTI samples. In Gram positive bacteria also, Tigecycline is the most sensitive antibiotic. The 2nd line drugs, Linezolid, vancomycin, Teicoplanin, are showing moderate level of sensitivity (around 70\%) even in OPD patients. Tetracyclines other than Tigecycline, Gentamycin, Fluoroquinolones and Macrolides have very poor senstivity in all categories (majority is $\leq 15 \%$ ). However, this whole pattern of different sensitivities in different categories was not found to be statistically significant ( $\mathrm{p}$ value $=0.0871$ )

\section{Discussion}

Vivekananda Polyclinic and Institute of Medical Sciences (VPIMS) is one of the prominent tertiary health care hospitals of North India. It is catering not only for the local patients of Lucknow but also for patients coming from adjacent peripheral areas as well as from far Nepal too. Majority of the patients are being referred with the history of receiving improper antibiotics treatment somewhere outside. The number of

Table: 1

Distribution of most Common Uropathogens (\%) in various categories.

\begin{tabular}{|c|c|c|c|c|c|c|c|c|}
\hline Uropathogens & $\begin{array}{l}\text { Isolates from OPD patients } \\
(\mathrm{N}=824)\end{array}$ & $(\%)$ & $\begin{array}{l}\text { Isolates from IPD } \\
\text { patients(N = 747) }\end{array}$ & $(\%)$ & $\begin{array}{l}\text { Isolates from Pediatric } \\
\text { patients }(\mathrm{N}=157)\end{array}$ & $(\%)$ & $\begin{array}{l}\text { Total positive isolates } \\
(\mathrm{N}=1728)\end{array}$ & $(\%)$ \\
\hline Escherichia coli & 545 & 66.1 & 313 & 41.9 & 66 & 42.0 & 924 & 53.4 \\
\hline Enterococcus spp. & 90 & 10.9 & 224 & 29.9 & 44 & 28.0 & 358 & 20.7 \\
\hline Klebsiellaspp. & 85 & 10.3 & 75 & 10.0 & 18 & 11.4 & 178 & 10.3 \\
\hline Candidaspp & 15 & 1.8 & 71 & 9.5 & 11 & 7.0 & 97 & 5.6 \\
\hline Acinetobacter spp. & 04 & 0.4 & 18 & 2.4 & 01 & 0.6 & 23 & 1.3 \\
\hline Pseudomonas aeruginosa & 07 & 0.8 & 14 & 1.8 & 05 & 3.1 & 26 & 1.5 \\
\hline Staphylococcus aureus & 07 & 0.8 & 02 & 0.2 & 00 & & 09 & 0.5 \\
\hline Proteus spp. & 17 & 2.0 & 00 & & 01 & 0.6 & 18 & 1.0 \\
\hline Sphingomonaspaucimobilis & 12 & 1.4 & 02 & 0.2 & 00 & & 14 & 0.8 \\
\hline Others & 42 & 5.0 & 28 & 3.7 & 11 & 3.8 & 110 & 6.3 \\
\hline
\end{tabular}




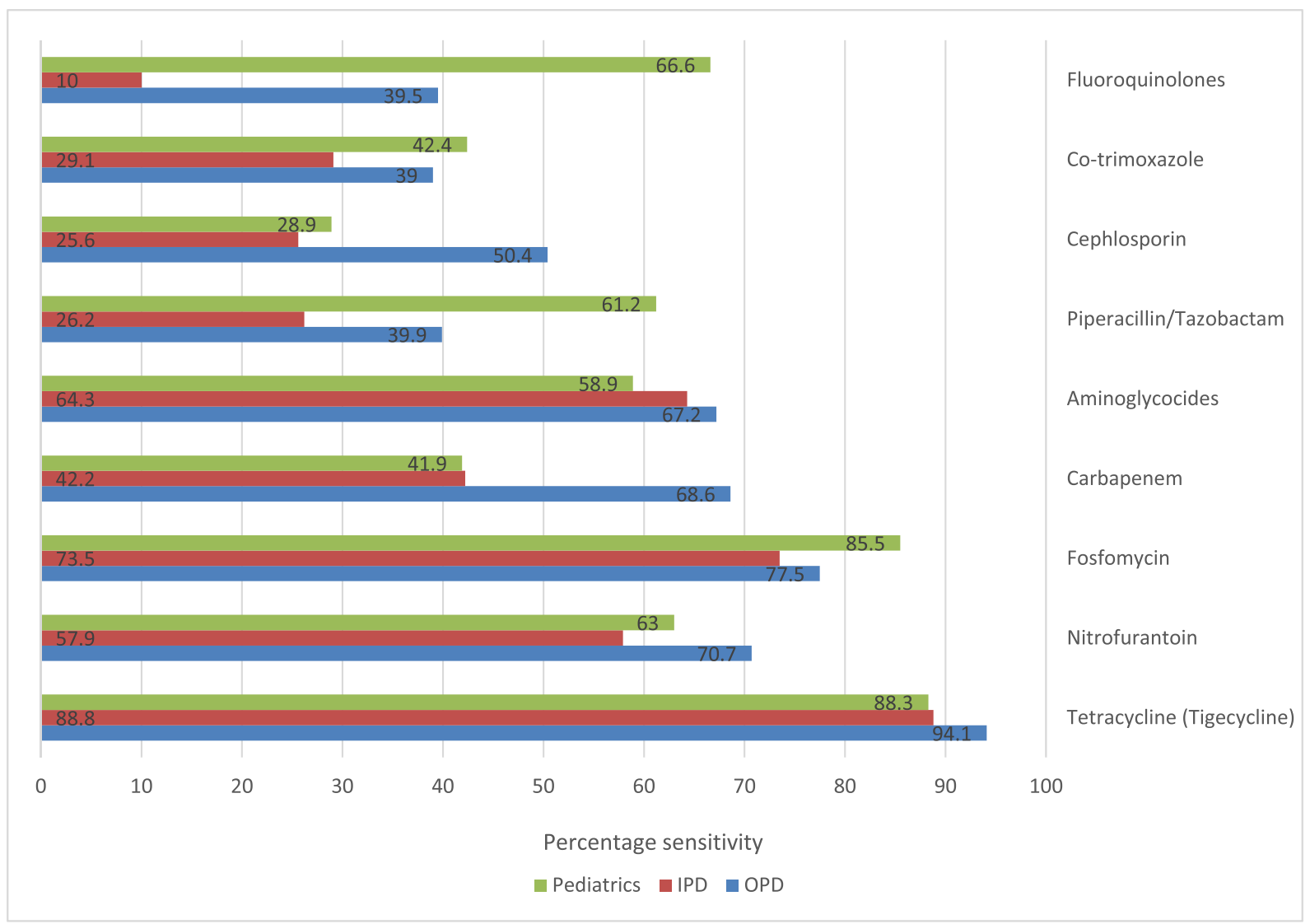

Fig. 1. Comparison of sensitivity pattern of Gram-negative bacilli in different groups of antibiotics among OPD, IPD and pediatrics patients.

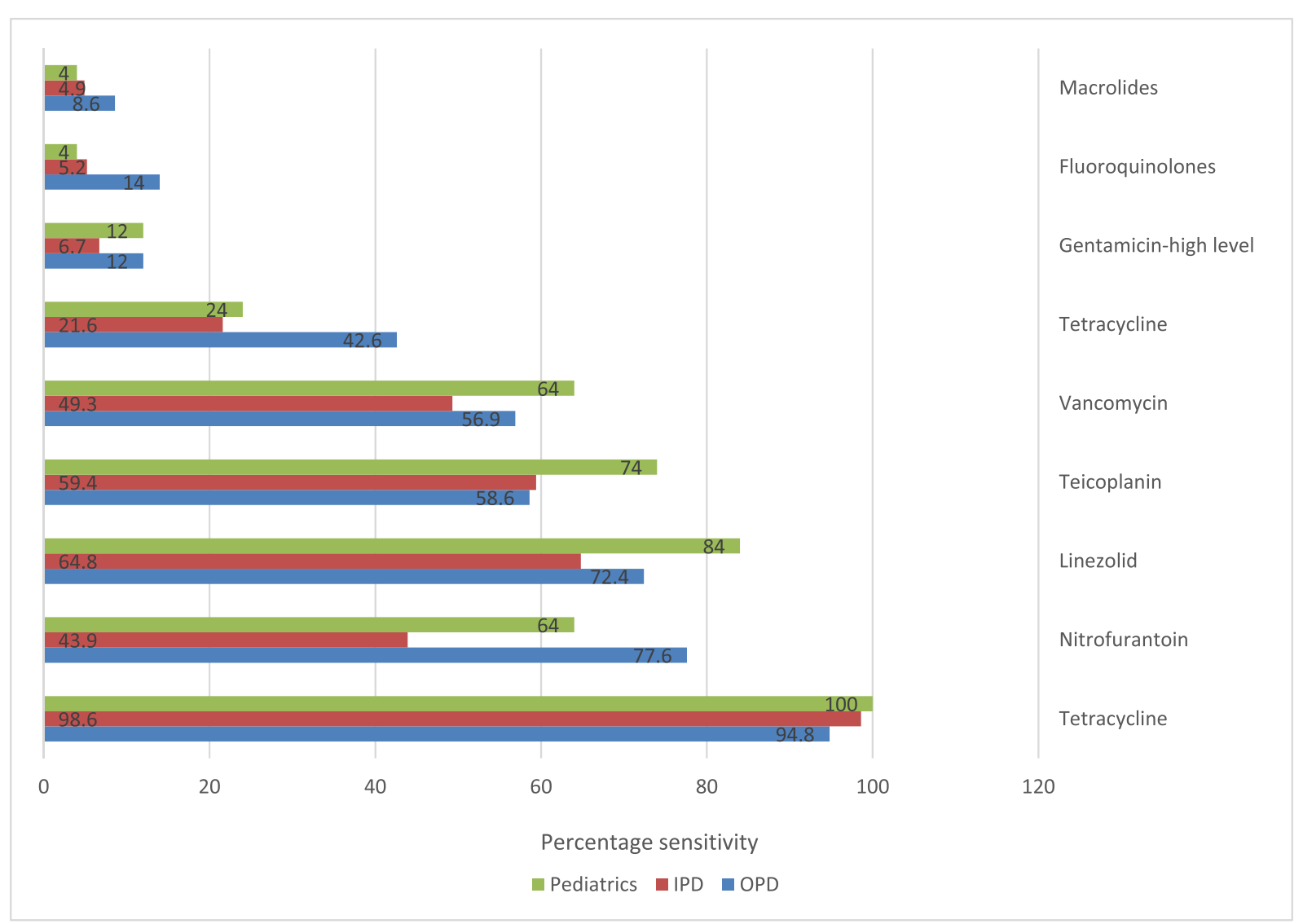

Fig. 2. Comparison of sensitivity pattern of most commonly isolated Gram-positive bacteria for different groups of antibiotics among OPD, IPD and Pediatric Patients. 
Table 2

Antibiotic sensitivity patterns of most commonly isolated Gram negative UTI isolates in OPD, IPD and Pediatrics patients

\begin{tabular}{|c|c|c|c|c|c|c|}
\hline \multirow[t]{2}{*}{ Group of Antibiotics studied } & \multicolumn{2}{|l|}{ OPD } & \multicolumn{2}{|l|}{ IPD } & \multicolumn{2}{|c|}{ Pediatric patients } \\
\hline & $\begin{array}{l}\text { E. coli (\%) } \\
\text { Sensitivity }\end{array}$ & $\begin{array}{l}\text { Klebsiella spp(\%) } \\
\text { Sensitivity }\end{array}$ & $\begin{array}{l}\text { E. coli (\%) } \\
\text { Sensitivity }\end{array}$ & $\begin{array}{l}\text { Klebsiella spp(\%) } \\
\text { Sensitivity }\end{array}$ & $\begin{array}{l}\text { E. coli (\%) } \\
\text { Sensitivity }\end{array}$ & $\begin{array}{l}\text { Klebsiella spp(\%) } \\
\text { Sensitivity }\end{array}$ \\
\hline Tetracycline (Tigecycline) & 95.2 & 95.3 & 79.5 & 94.5 & 81.0 & 95.6 \\
\hline Nitrofurantoin & 89.2 & 52.0 & 75.9 & 40.0 & 77.5 & 92.8 \\
\hline Fosfomycin & 86.3 & 58.3 & 75.0 & 72.0 & 72.8 & 70.6 \\
\hline Carbapenem & 82.1 & 56.2 & 52.4 & 32.0 & 68.9 & 57.1 \\
\hline Aminoglycosides & 74.7 & 58.3 & 50.9 & 42.0 & 52.4 & 59.7 \\
\hline Piperacillin/Tazobactam & 64.4 & 29.4 & 31.7 & 18.0 & 48.2 & 28.5 \\
\hline Cephalosporins & 60.7 & 39.5 & 35.3 & 16.0 & 20.6 & 34.2 \\
\hline Tetracycline (Other than Tigecycline) & 36.2 & 31.9 & 26.9 & 21.2 & 20.7 & 20.3 \\
\hline Co-trimoxazole & 28.8 & 54.2 & 18.2 & 40.0 & 48.2 & 35.7 \\
\hline Fluoroquinolones & 25.2 & 12.6 & 1.8 & 0.0 & 29.3 & 19.5 \\
\hline
\end{tabular}

partially treated or maltreated patients is significantly more. This is the condition of almost all tertiary level centers of North India.

Therefore, this present study was conducted to find out the present scenario of anti-microbial sensitivity in isolates causing UTI in patients referred to a tertiary care setup of North India. Out of total patients attending outdoor and indoor departments of VPIMS with symptoms suggestive of UTI, $16.9 \%$ had culture positivity, which is close to other studies too. A study in 2011 recorded positivity of $24 \%{ }^{13}$ and another study by BHN Yasmeen et al. ${ }^{14}$ in 2014 in Bangladesh showed $21 \%$ positivity in urine samples for pathogenic organisms. Similarity in positivity suggests that pathogenecity of resistant isolates is not affected; probably only the clearance of bacteria from host body is delayed.

In this study, the most predominant isolated organism was Escherichia coli as is seen in other studies too, but proportion (53.4\%) is lower in comparison to other studies. ${ }^{15}$ In contrast, proportion of Enterococcus spp (20.7\%) and Klebsiella spp (10.3\%) were higher in comparison to other studies. Probably, Enterococci and Klebsiella are more prevalent in our population partially replacing Escherichia coli population as uropathogen.

In this study, the most effective antibiotic, in vitro was Tigecycline for both Gram Positive and Gram-Negative isolates. However, its use in UTI is still controversial. There are few studies which advocate its use in case of nonavailability of other alternatives due to resistance or else. Therefore, authors suggest its cautious use. Uropathogens show higher degree of resistance against most of the antibiotics used for sensitivity including carbapenems. This increasing resistance may be due to improper dosing and duration of antibiotics in this particular region. ${ }^{16}$ Nitrofurantoin, one of the oldest oral antibiotics, showed high sensitivity. It showed its effectiveness against $70-75 \%$ of the isolates obtained from OPD. This drug exhibited low resistance rate in the major part of the world $(0-5.4 \%)$, despite of its use for many years which was because of localized action of this drug only on the urinary tract. ${ }^{17}$ So, nitrofurantoin can be considered as first line, cost saving and effective oral therapy in UTI patients at OPD level. However, at IPD level and in pediatric patients, its effectiveness is still questionable.

Significant reduction in sensitivity pattern was detected for carbapenem, cephalosporins, fluoroquinolones etc., possibly because of irrational use of these antibiotics in past few years with inadequate dose and duration. Uropathogens resistant to antibiotic have now become a public health concern in India. According to guidelines by Infectious Diseases Society of America (IDSA) in the year 2011, an antibiotic is no longer recommended for empirical treatment of acute UTI if there is $>20 \%$ resistance prevalence to that particular antibiotic recorded. ${ }^{18}$ Ironically, most of the antibiotics used in our study cannot be recommended for empirical treatment of acute UTI. Therefore, our standard treatment guidelines for UTI are not appropriate for the study population. A large-scale study and further amendments in policy are needs of the hour.

\section{Conclusion}

There is urgent need of constant monitoring with culture and sensitivity pattern of specific pathogens in different health care centers in our country. Community awareness program should be undertaken for adherence to treatment protocol considering bacterial resistance and emerging multidrug resistant strains. It is necessary to conduct a regional research on the culture and sensitivity patterns of the bacteria.

\section{References}

1. Schaeffer AJ. Infection of the urinary tract. Campbell's urology. 2002;1:515-602

2. Sleigh JD, Timbury MC. second ed.Notes on Medical Bacteriology. . New York: Churchill Livingstone Inc., 1560 Broadway; 1986:212-218.

3. Turck M, Stamm W. Am J Med. 1981 Mar;70(3):651-654.

4. Dias Neto JA, Dias Magalhaes da Silva L, Carlos Pereira Martins A, BrianeziTiraboschi R, Alonso Domingos AL, et al. Prevalence and bacterial susceptibility of hospital acquired urinary tract infections. Acta Cir Bras. 2003;18:36-38.

5. Beyene G, Tsegaye W. Bacterial uropathogens in urinary tract infections andantibiotic susceptibility pattern injimma university specialized hospital, southwest Ethiopia. Ethiopian $j$ health science. 2011;21:141-146.

6. Moges F, Genetu A. Antibiotic senstivity of common bacterial pathogens in urinarytract infection at Gonder Hospital, Ethiopia. East Afr Med J. 2002:79:140-142.

7. Farrell DJ, Morrissey I, De Rubeis D. A UK multicentre study of the antimicrobial susceptibility bacterial pathogens causing urinary tract infection. J Infect. 2003:46:94-100.

8. Mathai D, Jones RN, Pfaller MA. Epidemiology and frequency of resistance amongpathogenscausing urinary tract infections in 1,510 hospitalized patients: a report from the sentryantimicrobial surveillance program (North America). Diagn Microbiol Infect Dis. 2001:40:129-136, 20

9. Kunin CM. Detection, Prevention and Management of Urinary Tract Infections. vii. Philadelphia: Lea and Febiger; 1987:193.

10. Munoz-Dávila MJ, Roig M, Yagüe G. Eur J Clin Microbiol Infect Dis. 2013;32(6):77380 Epub 2013 Jan 12

11. Sleigh JD, Timbury MC. Notes on Medical Bacteriology. second ed. New York: Churchill Livingstone Inc., 1560 Broadway; 1986:212-218.

12. Performance CLSI. Standards for Antimicrobial Susceptibility Testing. 28 th ed Wayne, PA: Clinical and Laboratory Standards institute; 2018 CLSIsupplement M100.

13. Yasmeen B, Islam S, Islam S, Uddin MM, Jahan R. Prevalence of urinary tract infection, its causative agents and antibiotic sensitivity pattern: a study in Northern International Medical College Hospital, Dhaka. N. Int. Med. Coll. J. 2015;7(1):105-109.

14. Yasmeen BN, Islam S, Islam S, Uddin MM, Jahan R. Prevalence of urinary tract infection, its causative agents and antibiotic sensitivity pattern: a study in Northern International Medical College Hospital, Dhaka. N.Int. Med.Coll. J. 2015;7(1):105-109.

15. Niranjan V, Malini A. Antimicrobial resistance pattern in Escherchia coli causing urinary tract infection among inpatients. Indian J Med Res. 2014;139(6):945-948.

16. Majumder MI, Ahmed T, Sakib N, Khan AR, Saha CK. A follow up study bacteriology and antibiotic sensitivity pattern of urinary tract infection in a tertiary care hospital in Bangladesh. J Bacteriol Parasitol. 2018;9:334.

17. Prais D, Straussberg R, Avitzur Y, Nussinovitch M, Harel L, et al. Bacterialsusceptibility to oral antibiotics in community acquired urinary tract infection. Arch Dis Child. 2003;88:215-218.

18. Colgan R, Willams M. Diagnosis and treatment of acute uncomplicated cystitis. Am Fam Physician. 2011;84:771-776. 